TAMKANG JOURNAL OF MATHEMATICS

Volume 33, Number 4, Winter 2002

\title{
RINGS WITH GENERALIZED COMMUTATORS IN THE NUCLEI
}

\author{
Dedicated to my father on his 85th birthday
}

CHEN-TE YEN

\begin{abstract}
Let $R$ be a prime weakly Novikov ring and $T_{k}=\underbrace{[[[\ldots[[R, R], R] \ldots, R], R], R]}_{k R^{\prime} s}$ where
$k$ is a positive integer. We prove that if $T_{k} \subseteq N_{l} \cap N_{r}$ or $T_{k} \subseteq N_{m} \cap N_{r} R^{\prime} s$ or $T_{k}=0$. Moreover, if $T_{k}$ is contained in two of the three nuclei, and $k=2$ or $k=3$ then the same conclusions hold. We also consider such rings with derivations. Some similar results of weakly M-rings are obtained.
\end{abstract}

\section{Introduction}

Let $R$ be a nonassociative ring. We shall denote the associator and commutator by $(x, y, z)=(x y) z-x(y z)$ and $[x, y]=x y-y x$ for all $x, y, z$ in $R$ respectively. In any ring $R$, one has the following nuclei:

$$
\begin{aligned}
N_{l} & =\{n \in R \mid(n, R, R)=0\}-\text { left nucleus, } \\
N_{m} & =\{n \in R \mid(R, n, R)=0\}-\text { middle nucleus, } \\
N_{r} & =\{n \in R \mid(R, R, n)=0\}-\text { right nucleus. } \\
N & =N_{l} \cap N_{m} \cap N_{r}-\text { nucleus. }
\end{aligned}
$$

A ring $R$ is called simple if $R$ is the only nonzero ideal of $R$. Thus, $R^{2}=R$.

A ring $R$ is called semiprime if the only ideal of $R$ which squares to zero is the zero ideal. A ring $R$ is called prime if the product of any two nonzero ideals of $R$ is nonzero. Note that each associator and commutator are linear in each argument. Thus $N_{l}, N_{m}$ and $N_{r}$ are additive subgroups of $(R,+)$. If $S$ is a nonempty subset of a ring $R$, then the ideal of $R$ generated by $S$ is $\langle S\rangle$. A ring $R$ is called weakly Novikov [4] if $R$ satisfies the following identity.

$$
(w, x, y z)=y(w, x, z) \quad \text { for all } w, x, y, z \text { in } R .
$$

Received December 25, 2001.

2000 Mathematics Subject Classification. Primary 17A30, 17A36.

Key words and phrases. Nucleus, simple ring, semiprime ring, prime ring, weakly Novikov ring, derivation, associator ideal, weakly M-ring. 
An additive mapping $d$ on a ring $R$ is called a derivation if $d(x y)=d(x) y+x d(y)$ holds for all $x, y$ in $R$. For any ring $R$, let $T_{k}=\underbrace{[[[\ldots[[R, R], R] \ldots, R], R], R]}_{k R^{\prime} s}$ where $k$ is a positive integer. Note that $T_{2}=[R, R]$ and $T_{3}=[[R, R], R]$. We also note that $\left[R, T_{k}\right]=\left[T_{k}, R\right] \subseteq T_{k}$, where $k$ is a positive integer. Obviously, we have the following identities.

$$
\begin{aligned}
& T_{k}+T_{k} R=T_{k}+R T_{k} \quad \text { for all positive integers } k . \\
& d(R)+d(R) R=d(R)+R d(R) . \\
& d((x, y, z))=(d(x), y, z)+(x, d(y), z)+(x, y, d(z)) \quad \text { for all } x, y, z \text { in } R . \\
& S(x, y, z)=(x, y, z)+(y, z, x)+(z, x, y)=[x y, z]+[y z, x]+[z x, y] \\
& \text { for all } x, y, z \text { in } R .
\end{aligned}
$$

We shall use the Teichmüller identity

$$
(w x, y, z)-(w, x y, z)+(w, x, y z)=w(x, y, z)+(w, x, y) z \text { for all } w, x, y, z \text { in } R,
$$

which is valid in every ring.

As a consequence of (6), we have that $N_{l}, N_{m}$ and $N_{r}$ are associative subrings of $R$. Suppose that $n \in N_{l}$ Then with $w=n$ in (6) we obtain

$$
(n x, y, z)=n(x, y, z) \text { for all } x, y, z \text { in } R \text { and } n \text { in } N_{l} \text {. }
$$

Suppose that $m \in N_{r}$. Then with $z=m$ in (6) we get

$$
(w, x, y m)=(w, x, y) m \text { for all } w, x, y \text { in } R \text { and } m \text { in } N_{r} .
$$

Suppose that $j \in N_{l} \cap N_{m}$. Then with $x=j$ in (6) we have

$$
(w j, y, z)=(w, j y, z) \quad \text { for all } w, y, z \text { in } R \text { and } j \text { in } N_{l} \cap N_{m} .
$$

Definition 1. Let $A$ be the associator ideal of a ring $R$.

Ordinary by (6) $A$ can by characterized as all finite sums of associators and left multiples of associators. In view of (1) it suffices to take all finite sums of associators if $R$ is a weakly Novikov ring. Hence, in this case $A=(R, R, R)$. In the paper, we consider rings with generalized commutators in the nuclei. There had been other results concerning rings in which $[R, R] \subseteq N_{l}$. For example Thedy [5], Kleinfeld [1], Kleinfeld and Kleinfeld [2] as well as Kleinfeld and Smith [3].

Definition 2. For any ring $R$, let $V_{k}=T_{k}+R T_{k}$ for all positive integers $k$. 


\section{Results of Weakly Novikov Rings}

Lemma 1. If $R$ is a weakly Novikov ring, then $R N_{r} \subseteq N_{r}$ and $A \cdot N_{r}=(R, R, R)$. $N_{r}=0$.

Proof. Let $z \in N_{r}$ and $w, x, y \in R$. Then by (8) and (1), we have $(w, x, y) z=$ $(w, x, y z)=y(w, x, z)=0$. Thus, we get $A \cdot N_{r}=(R, R, R) \cdot N_{r}=0$ and $R N_{r} \subseteq N_{r}$, as desired.

By (2) and the result of [8], we have the

Lemma 2. If $R$ is a ring such that $T_{k}$ is contained in two of the three nuclei, then $V_{k}$ is an ideal of $R$ for every positive integer $k$.

In the sequel, for the convenience we denote $T_{k}$ and $V_{k}$ by $T$ and $V$ respectively.

Theorem 1. If $R$ is a prime weakly Novikov ring such that $T \subseteq N_{l} \cap N_{r}$ or $T \subseteq$ $N_{m} \cap N_{r}$, then $R$ is associative or $T=0$.

Proof. Using $T \subseteq N_{r}$ and Lemma 1, we get

$$
A \cdot V=A \cdot(T+R T)=0 .
$$

By Lemma 2 and the primeness of $R,(10)$ implies $A=0$ or $V=0$. Thus, $R$ is associative or $T=0$.

Lemma 3. If $R$ is a weakly Novikov ring such that $T \subseteq N_{l} \cap N_{m}$, then

$$
(R, R, T) R=0
$$

Proof. Note that $[R, T]=[T, R] \subseteq T$. Using this, the hypotheses, (6),(1),(9) and (7), for all $y \in T$, and $w, x, z \in R$ we have $(w, x, y) z=w(x, y, z)+(w, x, y) z=(w x, y, z)-$ $(w, x y, z)+(w, x, y z)=-(w,[x, y], z)-(w, y x, z)+y(w, x, z)=-(w y, x, z)+y(w, x, z)=$ $-([w, y], x, z)-(y w, x, z)+y(w, x, z)=0$, Hence, we get $(R, R, T) R=0$,as desired.

Theorem 2. Let $R$ be a prime weakly Novikov ring such that $T \subseteq N_{l} \cap N_{m}$. If $S(x, y, z) \in N_{m}$ for all $x, y, z$ in $R$, or $[T,(R, R, R)]=0$, then $R$ is associative or $T=0$.

Proof. Assume that $S(x, y, z) \in N_{m}$ for all $x, y, z$ in $R$. Using this, (5) and the hypotheses, for all $x \in T$ and $y, z \in R$ we get $(y, z, x)=(x, y, z)+(y, z, x)+$ $(z, x, y)=S(x, y, z) \in N_{m}$. Thus, $(R, R, T) \subseteq N_{m}$. Applying this, (1) and (11), we have $(R, R, R T) R=R(R, R, T) \cdot R=R \cdot(R, R, T) R=0$.

Combining this with (11) results in

$$
(R, R, V) R=0 .
$$

Assume that $[T,(R, R, R)]=0$. Using this, $(1),(11)$ and $(6)$, and noting that $[T, R] \subseteq$ $T$, for all $w, x, y, t \in R$, and $z \in T$ we have $(w, x, y) z \cdot t=z(w, x, y) \cdot t=(w, x, z y) t=$ 
$(w, x,[z, y]) t+(w, x, y z) t=w(x, y, z) \cdot t+(w, x, y) z \cdot t+(w, x y, z) t-(w x, y, z) t=w(x, y, z) \cdot$ $t+(w, x, y) z \cdot t$ and so $(x, y, w z) t=w(x, y, z) \cdot t=0$. Combining this with (11), we also obtain (12).

Using (1) and (12), we see that $\langle(R, R, T)\rangle=(R, R, V)$. By the semiprimeness of $R$, (12), implies $(R, R, V)=0$. Hence, $V \subseteq N_{r}$

Consequently, $T \subseteq N$. By Theorem $1, R$ is associative or $T=0$.

In [3], Kleinfeld and Smith had proved that if $R$ is a prime left alternative ring with $[R, R] \subseteq N_{l}$ and characteristic $\neq 2,3$ then $R$ is associative. A linearization of the left alternative identity shows that $N_{l}=N_{m}$. We have the similar result for the weakly Novikov ring case.

Theorem 3. If $R$ is a prime weakly Novikov ring such that $[R, R]$ is contained in two of the three nuclei, then $R$ is associative or commutative.

In the latter case, $N_{r}=0$ or $R$ is associative.

Proof. In view of Theorem 1, we may assume $[R, R] \subseteq N_{l} \cap N_{m}$. Let $B=[R, R]+$ $R[R, R]$. By Lemma $2, B$ is an ideal of $R$. Using Lemma 3 , we get

$$
(R, R,[R, R]) R=0 .
$$

Applying (5) and $[R, R] \subseteq N_{l} \cap N_{m}$, for all $x, y, z \in R$ we have $S(x, y, z)=(x, y, z)+$ $(y, z, x)+(z, x, y) \in N_{l} \cap N_{m}$. Let $x \in[R, R]$. Then we get $(y, z, x) \in N_{l} \cap N_{m}$. Thus, we obtain $(R, R,[R, R]) \subseteq N_{l} \cap N_{m}$. Using this and (13), we have $R(R, R[R, R]) \cdot R=$ $R \cdot(R, R,[R, R]) R=0$.

Hence, applying this, (1) and (13), and noting that $B$ is an ideal of $R$, we obtain that $(R, R, B) \cdot R=0$ and $\langle(R, R,[R, R])\rangle=(R, R, B)$. Thus, by the semiprimeness of $R$ we get $(R, R, B)=0$ and so $[R, R] \subseteq N_{r}$. By Theorem $1, R$ is associative or commutative.

Assume that $R$ is commutative. Thus we have $N_{r} R=R N_{r} \subseteq N_{r}$ and $A \cdot N_{r}=0$ by Lemma 1. Hence $N_{r}$ is an ideal of $R$. By the primeness of $R, A \cdot N_{r}=0$ implies $A=0$ or $N_{r}=0$.

By Theorem 3, we obtain the

Corollary 1. If $R$ is a prime weakly Novikov ring such that $[R, R]$ is contained in two of the three nuclei with $N_{r} \neq 0$ or $[R, R] \neq 0$, then $R$ is associative, that is $N_{r}=R$.

In the sequel, for the convenience we denote $V_{3}$ by $D$.

Lemma 4. If $R$ is a weakly Novikov ring such that $[[R, R], R] \subseteq N_{l} \cap N_{m}$ then $\langle(R, R, D)\rangle \cdot(R, R, R)=0$, where $\langle(R, R, D)\rangle=(R, R, D)+(R, R, D) R+R$. $(R, R, D) R$.

Proof. Let $D=[[R, R], R]+R[[R, R], R]$ and $[[R, R], R] \subseteq N_{l} \cap N_{m}$. By Lemma 3 , we obtain

$$
(R, R,[[R, R], R]) R=0 .
$$

Thus (14) implies

$$
(R, R,[[R, R], R]) \subseteq N_{l} .
$$


Assume that $y \in[[R, R], R]$ and $w, x, z, u, v, t \in R$. Using (14), the hypotheses and (5) we have $z(w, x, y)=[z,(w, x, y)]=[z, S(w, x, y)] \in[[R, R], R] \subseteq N_{l} \cap N_{m}$ and so by (1) twice we get $(w, x,[z, y])+y(w, x, z)=(w, x,[z, y])+(w, x, y z)=(w, x, z y)=z(w, x, y) \in$ $N_{l} \cap N_{m}$. Applying these, (1) and (15) we obtain the following two inclusions.

$$
\begin{aligned}
& (R, R, R[[R, R], R])=R(R, R,[[R, R], R]) \subseteq N_{l} \cap N_{m} . \\
& {[[R, R], R] A=[[R, R], R](R, R, R) \subseteq N_{l} .}
\end{aligned}
$$

Then (17) implies

$$
[[R, R], R] A \cdot R=[[R, R], R] \cdot A R \subseteq[[R, R], R] A \subseteq N_{l} .
$$

Combined (15) with (16) results in

$$
(R, R, D) \subseteq N_{l}
$$

Using (1), (17), (7) and (18), we have $(w, x, y z)(u, v, t)=y(w, x, z) \cdot(u, v, t)=(y(w, x, z)$. $u, v, t)=0$. Hence applying this, (2) and (14) we obtain

$$
(R, R, D) A=(R, R, D)(R, R, R)=0 .
$$

Then by (20), (19), (7) and (1) we get $0=(R, R, D)(R, R, R)=((R, R, D) R, R, R)$ and $0=(R, R, D)(R, R, R)=(R, R,(R, R, D) R)$. Thus, by these, (14) and (1) we have

$$
R(R, R,[[R, R], R]) \cdot R=(R, R, D) R \subseteq N_{l} \cap N_{r} .
$$

Let $x \in R(R, R,[[R, R], R])$ and $w, y, z \in R$. Then by (16) and (1) we get $x \in N_{l} \cap N_{m}$ and $w x \in(R, R, D)$. Hence by (9) and (19), we obtain $(w, x y, z)=(w x, y, z)=0$. Combined this, (1), (14) and (21) results in

$$
(R, R, D) R \subseteq N .
$$

Using (1), (19) and (22) we see that $\langle(R, R, D)\rangle=(R, R, D)+(R, R, D) R+R$. $(R, R, D) R$.

Combined (19) with (20) results in

$$
(R, R, D) R \cdot A=(R, R, D) \cdot R A \subseteq(R, R, D) A=0 .
$$

Apping (22) and (23), we get $\{R \cdot(R, R, D) R\} \cdot A=R \cdot\{(R, R, D) R \cdot A\}=0$. Thus using this, (20) and (23), we have $\langle(R, R, D)\rangle \cdot A=0$, as desired.

Theorem 4. If $R$ is a prime weakly Novikov ring such that $[[R, R] R]$ is contained in two of the three nuclei, then $R$ is associative of $[[R, R], R]=0$.

Proof. In view of Theorem 1, we may assume $[[R, R], R] \subseteq N_{l} \cap N_{m}$. Let $D=[[R, R], R]+R[[R, R], R]$. Then by Lemma 4 we obtain $\langle(R, R, D)\rangle \cdot A=0$, where $\langle(R, R, D)\rangle=(R, R, D)+(R, R, D) R+R \cdot(R, R, D) R$. By the semiprimeness 
of $R$, this implies $\langle(R, R, D)\rangle=0$. Hence $[[R, R], R] \subseteq N_{r}$. Thus by Theorem $1, R$ is associative or $[[R, R], R]=0$.

By Theorem 4, we have the

Corollary 2. If $R$ is a prime weakly Novikov ring such that $[[R, R], R]$ is contained in two of the three nuclei with $[[R, R], R] \neq 0$, then $R$ is associative.

The following is very easy.

Remark 1. If $R$ is a simple weakly Novikov ring such that $T \subseteq N_{r}$, then $R$ is associative or $T=0$.

Proof. Assume that $R=A=(R, R, R)$. By Lemma 1, we have $R T=A T=0$. Thus, we get $T R=[T, R] \subseteq T$. Hence, we see that $\langle T\rangle=T$. By the simplicity of $R$, we obtain $T=0$, as desired.

Remark 2. If $R$ is a semiprime weakly Novikov ring such that $(R, R, R) \subseteq N_{l}$ or $(R, R, R) \subseteq N_{r}$ then $R$ is associative.

Proof. We see that the associator ideal $A$ of $R$ is all finite sums of associators. Assume that $(R, R, R) \subseteq N_{l}$. Then by this and (7), for all $w \in(R, R, R)$ and $x, y, z \in R$ we get $w(x, y, z)=(w x, y, z) \in(A, R, R)=0$.

Thus, we have $(R, R, R)(R, R, R)=0$ and so $A^{2}=0$.

Assume that $(R, R, R) \subseteq N_{r}$. Then by Lemma 1 , we obtain

$$
(R, R, R)(R, R, R)=(R, R, R(R, R, R))=(R, R, A)=0
$$

In either case, we have $A^{2}=0$. By the semiprimeness of $R$, this implies $A=0$. Thus, $R$ is associative.

In view of Theorem 1 of [6], we have the

Remark 3. If $R$ is a semiprime weakly Novikov ring with a derivation $d$ such that $d(R) \subseteq N_{r}$, then $d(A)=0$. Moreover, if $R$ is prime such that $d(R) \subseteq N_{l} \cap N_{r}$ or $d(R) \subseteq N_{m} \cap N_{r}$, then $R$ is associative or $d=0$.

Proof. By the definition of $d, d(R) \subseteq N_{r},(8),(1)$ and $A=(R, R, R)$, for all $w, x, y, z, t \in R$ we get $(w, x, y) d(z)=(w, x, y d(z))=y(w, x, d(z))=0,(w, x, y) \cdot \operatorname{td}(z)=$ $(w, x, y) t \cdot d(z)=0$ and so $d(y)(w, x, z)=(w, x, d(y) z)=(w, x, d(y) z)+(w, x, y d(z))=$ $(w, x, d(y z))=0$.

Let $E=d(R)+R d(R)$. Then the above three equalities imply

$$
A \cdot E=0 \text { and } d(R) \cdot A=0 .
$$

Using (24), we have that $d(A) R \subseteq d(A)$ and $R d(A) \subseteq d(A)$. Hence $\langle d(A)\rangle=d(A)$. Applying (4), we see that $d(A) \subseteq A$. Thus by $(24), d(A) \cdot A=0$ and so by the semiprimeness of $R$, this implies $d(A)=0$. 
Assume that $R$ is prime such that $d(R) \subseteq N_{l} \cap N_{r}$ or $d(R) \subseteq N_{m} \cap N_{r}$. Then by (3) and the result of [8], $E$ is an ideal of $R$. By the primeness of $R,(24)$ implies $A=0$ or $E=0$. Hence, $R$ is associative or $d=0$.

In Remark 3, if $R$ is a semiprime weakly Novikov ring with a derivation $d$ such that $d(R) \subseteq N_{r}$, then $d(A)=0$. Hence, the results of [7] can be applied.

\section{Results of weakly M-rings}

In the sequel, we denote $T_{k}$ and $V_{k}$ by $T$ and $V$ respectively.

A ring $R$ is called a weakly M-ring if $R$ satisfies the following identity.

$$
(w, x y, z)=x(w, y, z) \text { for all } w, x, y, z \text { in } R .
$$

Note that if $R$ is a weakly M-ring then by (6) and (25) we obtain $A=(R, R, R)$.

Theorem 5. If $R$ is a prime weakly M-ring such that $T \subseteq N_{l} \cap N_{m}$ or $T \subseteq N_{m} \cap N_{r}$, then $R$ is associative or $T=0$.

Proof. Note that $[T, R] \subseteq T$. Using this, $T \subseteq N_{m}$ and (25), for all $x \in T$ and $w, y, z, t \in R$ we have $x(w, y, z)=x(w, y, z)-y(w, x, z)=(w, x y, z)-(w, y x, z)=$ $(w,[x, y], z)=0$, and so $t x \cdot(w, y, z)=t \cdot x(w, y, z)=0$. These two identities yield

$$
V \cdot A=0
$$

Since $V$ is an ideal of $R$, by the primeness of $R,(26)$ implies $A=0$ or $V=0$. Hence, $R$ is associative or $T=0$.

The following three remarks are similar to those in section 2. The proofs are also similar, so we omit it.

Remark 4. If $R$ is a simple weakly M-ring such that $T \subseteq N_{m}$, then $R$ is associative or $T=0$.

Remark 5. If $R$ is a semiprime weakly M-ring such that $(R, R, R) \subseteq N_{m}$, then $R$ is associative.

Remark 6. If $R$ is a prime weakly M-ring with a derivation $d$ such that $d(R) \subseteq$ $N_{l} \cap N_{m}$ or $d(R) \subseteq N_{m} \cap N_{r}$, then $R$ is associative or $d=0$.

Finally, we ask if the theorem or the remark is valid for the other cases.

\section{References}

[1] Erwin Kleinfeld, Rings with $(x, y, x)$ and commutators in the left nucleus, Comm. Algebra 16(1988), 2023-2029.

[2] Erwin Kleinfeld and Margaret Kleinfeld, On a class of Lie admissible rings, Comm. Algebra 13(1985), 465-477. 
[3] Erwin Kleinfeld and Harry F. Smith On simple rings with commutators in the left nucleus, Comm. Algebra 19(1991), 1593-1601.

[4] Erwin Kleinfeld and Harry F. Smith, Semiprime flexible weakly Novikov rings are associative, Comm. Algebra 23(1995), 5077-5083.

[5] A. Thedy, Rings with commutators in the nuclei, Math. Zeitschr. 119(1971), 213-218.

[6] C. T. Yen, Rings with a derivation whose image is contained in the nuclei, Tamkang J. Math. 25(1994), 301-307. MR. 96e:17005. Zbl. 824. 17001.

[7] C. T. Yen, Rings with a derivation whose image is zero on the associators, Tamkang J. Math. 26(1995), 5-8. MR. 96e:17006. Zbl. 829. 17003.

[8] C. T. Yen and Irvin R. Hentzel, The ideal generated by sets contained in the nuclei, soochow J. Math. 22(1996), 435-438. MR. 1406 516. Zbl. 0858. 17002.

Department of Mathematics, Chung Yuan Christian University, Chung Li, Taiwan, 320, Republic of China. 